\title{
Shape-Based Image Retrieval Using $k$-Means Clustering and Neural Networks
}

\author{
Xiaoliu Chen and Imran Shafiq Ahmad ${ }^{\star}$ \\ School of Computer Science \\ University of Windsor \\ Windsor, ON N9B 3P4 - Canada \\ xiaoliuchen@yahoo.com, imran@cs.uwindsor.ca
}

\begin{abstract}
Shape is a fundamental image feature and belongs to one of the most important image features used in Content-Based Image Retrieval. This feature alone provides capability to recognize objects and retrieve similar images on the basis of their contents. In this paper, we propose a neural network-based shape retrieval system in which moment invariants and Zernike moments are used to form a feature vector. $k$ means clustering is used to group correlated and similar images in an image collection into $k$ disjoint clusters whereas neural network is used as a retrieval engine to measure the overall similarity between the query and the candidate images. The neural network in our scheme serves as a classifier such that the moments are input to it and its output is one of the $k$ clusters that has the largest similarity to the query image.
\end{abstract}

Keywords: image retrieval, shape-based image retrieval, $k$-means clustering, moment-invariants, Zernike moments.

\section{Introduction}

Recent advances in image acquisition, storage, processing, and display capabilities have resulted in more affordable and widespread use of digital images. As a result, there is an increased demand for effective management of image data. Given the huge amount of image data that exist now and will be collected in near future, the traditional approach of manual annotations is not only inadequate but also fails to serve the purpose. To utilize the image information efficiently, there is a constant demand for effective techniques to store, search, index and retrieve images based on their contents [1. This has led to a great emphasis and demand on the use of automatically extractable and mathematically quantifiable visual image features such as color, texture, shape, and spatial relationships. Such retrievals are generally termed as Content-based Image Retrieval (CBIR).

Shape is a very powerful and one of the fundamental image features that facilitates representation of image contents. Retrieval of images based on the shapes of objects, generally termed as Shape-based Retrieval, is an important

* Corresponding author. Authors would like to acknowledge partial support provided by the Natural Sciences and Engineering Research Council (NSERC) of Canada. 
CBIR technique and has applications in many different fields. Examples of such type of retrievals can be found but are not limited to recognition and retrieval of trademarks, logos, medical structures, fingerprints, face profiles, hand written signatures, etc. In many situations, people can recognize an object only with its shape. Most often, shape of an object can be obtained by traversing its boundary.

Primary issues associated with shape-based image retrieval are: shape representation, similarity measure and retrieval strategy. Shape representations are formalistic identification models of original shapes so that the important characteristics of shapes are preserved [2]. The goal of shape representation is to derive a numeric shape descriptor or a feature vector that can uniquely characterize the given shape. Two-dimensional shapes can be represented in a number of different ways. Generally, such methods can be divided into two main categories: (i) contour-based methods and (ii) region-based methods. In contour-based methods, emphasis is on the outer closed curve that surrounds the shape whereas in region-based methods, entire shape region occupied by the shape within a closed boundary on the image plane is considered. moment invariants [3] and Zernike moments 4 are examples of such methods.

This paper provides an efficient mechanism for indexing and shape-based retrieval of images from an image database. We analyze and compare performance of two region-based methods, viz., moment invariants and Zernike moments to derive feature descriptors for shape representation and image retrieval while using artificial neural network as an intelligent search engine instead of traditional techniques of multidimensional indexing trees. $k$-means clustering is employed to provide learning samples to the neural network to facilitate back-propagation training. A user can query the system using query-by-example approach. The moments of the objects in a query are computed on-the-fly and compared against those of the already indexed database images to retrieve similar images. The important contributions of our shape matching and retrieval approach can be summarized as:

- the proposed method is independent of geometric transformations, i.e., scale, translation and rotation of the shape of the query or the database images.

- images are classified using their moments and placed in $k$ number of distinct clusters such that images in the same cluster exhibit higher levels of correlation whereas it is low between images in different clusters. Therefore, by controlling $k$, to a great extent, one could possibly control the degree of similarity among various images in a cluster.

- although use of moment invariants is not new 35], our scheme provides a mechanism for efficient retrieval of stored images through an artificial neural network which in our scheme serves as an intelligent search engine.

Remainder of this paper is organized as follows: in Section 2, we provide a summary of shape representation techniques. Section 3 describes the proposed methodology to represent, classify and retrieve shape images. Results of our experiments are presented in Section 4 whereas Section 5 provides our conclusions. 


\section{Shape Representation}

Shape representations are formalistic identification models of original shapes so that their important characteristics are preserved 2. The goal of shape representation is to derive a set of numeric shape descriptors or a feature vector that can uniquely characterize a given shape. $2 \mathrm{D}$ shapes can be described in a number of different ways with several different classifications [2]. The most popular and widely used among them is the one proposed by Pavlidis [6]. It is based on the use of shape boundary points as opposed to the interior features of a shape.

\subsection{Moment Invariants}

Moment invariants are extensively used in the area of pattern recognition, shape representation and for shape similarity measures. Moment invariants are derived from moments of shapes and are unchanged under translation, scale and rotation [3].

$$
m_{p q}=\int_{x} \int_{y} x^{p} y^{q} f(x, y) d y d x
$$

The theory of moments provides an interesting and useful alternative to a series of expansions for representing a real bounded function [9]. Suppose $f(x, y)=0$ is such a $2 \mathrm{D}$ function on a region $R$, then the geometric moments of order $p+q$, for $p, q=0,1,2, \ldots$ are given by Equation (11). The uniqueness theorem [5] states that if $f(x, y)$ is piecewise continuous and has nonzero values only in a finite part of the $x y$-plane, moments of all orders exist and the moment sequence $m_{p q}$ is uniquely determined by $f(x, y)$.

Many shape features such as total mass (area), centroid, angle of the principal axis, bounding box, best-fit ellipse and eccentricity can be conveniently represented in terms of geometric moments [7. Moment invariants are usually specified in terms of centralized moments $\mu_{p q}$, i.e., the moments measured with respect to the center of mass $(\bar{x}, \bar{y})$ and are defined as:

$$
\mu_{p q}=\int_{x} \int_{y}(x-\bar{x})^{p}(y-\bar{y})^{q} f(x, y) d y d x
$$

where $\bar{x}=\frac{m_{10}}{m_{00}}, \bar{y}=\frac{m_{01}}{m_{00}}$ is the center of mass and the normalized moments are defined as [8]:

and

$$
\eta_{p q}=\frac{\mu_{p q}}{\mu_{00}^{\gamma}}
$$

$$
\gamma=\frac{p+q}{2}+1
$$

Once computed, the feature vector consists of seven components and can be used to index shapes in the image database. The values of the computed geometric moments are usually small but the values of higher order moment invariants in some cases are close to zero. Therefore, all of the moment invariants need to be further normalized into $[0,1]$ by the limit values of each dimension $[9]$. 


\subsection{Zernike Moments}

Based on the idea of replacing the conventional kernel of moments with a general transform, orthogonal moments have been proposed to recover the image from moments 10. Zernike moments are orthogonal moments and allow independent moments to be constructed to an arbitrarily high order. The complex Zernike moments are derived from the Zernike polynomials:

$$
V_{n m}(x, y)=V_{n m}(\rho \cos \theta, \rho \sin \theta)=R_{n m}(\rho) \exp (j m \theta)
$$

and

$$
R_{n m}(\rho)=\sum_{s=0}^{(n-|m|) / 2}(-1)^{s} \frac{(n-s) !}{s !\left(\frac{n+|m|}{2}-s\right) !\left(\frac{n-|m|}{2}-s\right) !} \rho^{n-2 s} .
$$

where

$n$ is a non-negative integer.

$m$ is an integer, subject to the conditions $n-|m|=$ even, and $|m| \leq n$.

$\rho=\sqrt{x^{2}+y^{2}}$ is the radius from $(x, y)$ to the image centroid.

$\theta=\tan ^{-1}\left(\frac{y}{x}\right)$ is the angle between $\rho$ and the $x$-axis.

Zernike polynomials are a complete set of complex-valued functions orthogonal over the unit circle, i.e., $x^{2}+y^{2}=1$ and the complex Zernike moments of order $n$ with repetition $m$ are defined as:

$$
A_{n m}=\frac{n+1}{\pi} \iint_{x^{2}+y^{2} \leq 1} f(x, y) V_{n m}^{*}(\rho, \theta) d x d y .
$$

where $*$ denotes the complex conjugate.

The precision of shape representation depends on the number of moments truncated from the expansion. Since Zernike basis functions take the unit disk as their domain, this disk must be specified before moments can be calculated. The unit disk is then centered on the centroid of the shape, thus, making the obtained moments scale and translation invariant 4. Rotation invariance is achieved by using only the magnitudes of the moments. The magnitudes are then normalized into $[0,1]$ by dividing them by the mass of the shape.

Zernike moments do not need to know the boundary information, thus, making them suitable for more complex shape representation. Zernike moments can be obtained for some arbitrary order, thus, eliminating the drawback of moment invariants in which higher order moments are difficult to construct 9. However, Zernike moments lose the important perceptual meaning as reflected in the moment invariants [9].

In our implementation, all the shapes are normalized into a unit disk of fixed radius of $32\left(2^{5}\right)$ pixels, i.e., $R=32$. The disk radius can also be 8,16 , or 64 but the larger the disk, more are the computations required but more details the feature will reflect. The unit disk is then centered on the shape centroid by the transformations we have discussed earlier. For digital images, we substitute the 
integrals with summations. As a result, the complex Zernike moments of order $n$ with repetition $m$ are defined as:

$$
A_{n m}=\frac{n+1}{\pi} \sum_{i} f(x, y) V_{n m}^{*}(\rho, \theta), x_{i}^{2}+y_{i}^{2} \leq R^{2} .
$$

For binary images, $f(x, y)$ can be replaced by $u(x, y)$. As mentioned earlier, Zernike transformation makes the obtained moments scale and translation invariant whereas rotation invariance is achieved by only using the magnitudes (absolute values) of the moments.

\section{Proposed Approach}

In the proposed scheme, we consider both the moment invariants and the Zernike moments to represent shape in an image. Even though both of these representations are region-based shape representations, we still need to determine boundary sequence of the shape object. In many computer vision applications, for simplicity and speed, use of binary shape representations is a common practice. Therefore, we assume that all the images are binary images. This assumption will not effect the system's performance since we are concerned only with the shape features of the images. We also assume that all the pixels in the object have a value of ' 1 ' and all the pixels in the background have a value of ' 0 '. Therefore, a boundary sequence [1] is a list of connected pixels on the edge of the object, separating the shape region and the background.

$$
u(x, y)=\left\{\begin{array}{l}
1, \text { if }(x, y) \in R \\
0, \text { otherwise }
\end{array}\right.
$$

From the definition of geometric moments in Equation (1), if $f(x, y) \geq 0$ is a $2 \mathrm{D}$ real bounded function, the $(p+q)^{t h}$ order moment of the shape enclosed by $f(x, y)$ is given in Equation (7). The binary function given in Equation (7) is a simple representation of a binary region $R$. By replacing $f(x, y)$ by $u(x, y)$ in Equation (11), we get the moments of the region $R$ and the Equation (1) becomes:

$$
m_{p q}=\iint_{R} x^{p} y^{q} d y d x
$$

For geometric moments of a digital binary image, the double integrals can be approximated by double summations and Equation (8) can be written as:

$$
m_{p q}=\sum_{x} \sum_{y} x^{p} y^{q}
$$

A similar procedure can be followed for Zernike moments. The above equations involve a large number of multiplications and additions. In real-time applications, 
the speed of computation is crucial to the system performance. For computational speedup in binary shapes, the Delta method [12] uses contributions from each row rather than the individual pixels, requiring only the coordinates of the first pixel and the length of the chained pixels of each row for the shape $R$. The Integral method [7], on the other hand, uses contribution of each extreme point of the shape in each row instead of the contribution of the total row. Computations in this case are directly based on the integral operation. The geometric moments and both moment invariants and Zernike moments in this method are derived from the chain codes.

Based on the idea of computing from the integral and extreme pixels, moments are calculated by the following method. Let a given shape is considered a matrix of pixels then if $x_{L, i}, x_{R, i}$ are the abscissas of the first pixel (extreme left) and the last pixel (extreme right) of the shape in row $i, \delta_{i}$ is the number of connected pixels in row $i$, i.e., $\delta_{i}=x_{L, i}-x_{R, i}+1, y_{i}$ is the ordinate of row $i$, the geometric moments $m_{p q}$ can be written as $m_{p q}=\sum_{i} m_{p q, i}$.

The contribution of row $i$ in terms of $x_{L, i}, x_{R, i}$ and $y_{i}$ for a horizontal convex shape is considered as a region consisting of small uniform squares of size $1 \times 1$. Therefore, we adjust the coordinates by $\pm 1 / 2$ and the contribution of row $i$ is derived using the Newton-Leibniz formula such that the $(p+q)^{t h}$ moment of the whole binary shape is the sum of the contributions of every row $m_{p q}=\sum_{i} m_{p q, i}$. For a digital binary shape, the central moments are given as:

$$
\mu_{p q}=\sum_{x} \sum_{y}(x-\bar{x})^{p}(y-\bar{y})^{q}
$$

After $m_{p q}$ have been calculated by the extreme pixels, central moments $\mu_{p q}$ can be obtained [8].

\subsection{Indexing and Retrieval Approach}

Traditional indexing in CBIR involves use of multidimensional indexing trees to manage extracted visual features. In our approach, clustering and neural network is used to organize images and to build an intelligent retrieval engine.

Our strategy consists of two stages: training and testing, i.e., retrieval. The overall system architecture is presented in Fig[1. In the training stage, we use all of the images in our image database as training samples. We first group the training images into clusters using their feature vectors and then train the neural network with the results of clustering. In the testing stage, for a query image $q$, we use same technique to extract its features to build a feature vector which then becomes an input to the trained neural network for retrieval. The network assigns it to one or more similar clusters. We compare all of the images in the selected cluster(s) against the query image $q$. The distance between the query image and the database images is determined using a distance function, to be explained in Section 3.3 . Finally, similar images are ranked by their similarities and returned as the retrieval results. 


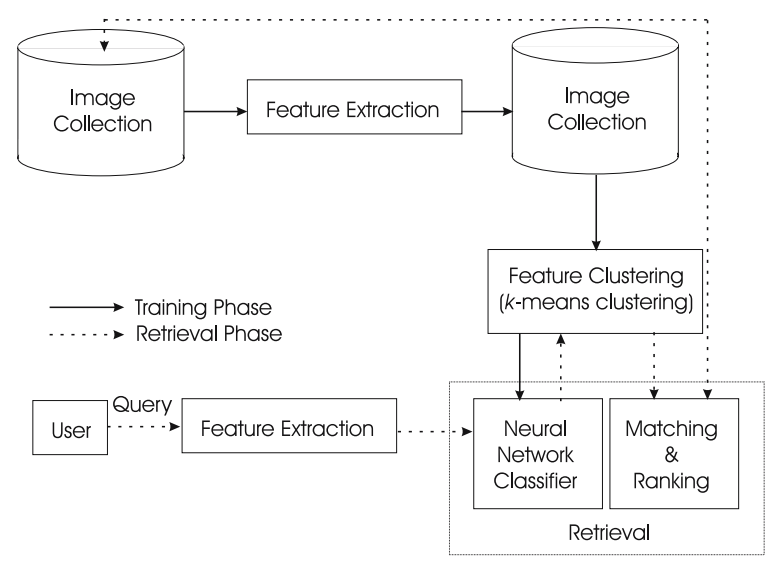

Fig. 1. Architecture of the proposed system

\subsection{Clustering}

$k$-means clustering [26] is one of the best known non-hierarchical clustering algorithm in pattern recognition. For a specified number of clusters $k$, the scheme assigns each input sample to one of the $k$ clusters so as to minimize the dispersion within the clusters. The membership for every sample is determined through an unsupervised learning procedure. The algorithm of $k$-means clustering first randomly initializes $k$ cluster means and then assigns each pattern vector to the cluster with nearest mean. It re-computes the cluster means and then re-assigns pattern vectors until no further changes occur. The nearest mean $\mu_{i}$ is found by using an arbitrary distance function. Initial values of $\mu_{i}$ affect the convergence since different initialization values may lead to different membership results. Therefore, we either guess initial value based on the knowledge of the problem or choose $k$ random samples from the data set $\left\{x_{1}, x_{2}, \ldots, x_{n}\right\}$.

Clustering techniques generally aim to partition the data set into a number of clusters. In our approach, our aim is to use a clustering algorithm that could provide us a minimum within-cluster distance and maximum inter-cluster distance. Based on this idea, there are several cluster validity indices to evaluate partitions obtained by the clustering algorithm, including Davies-Bouldin (DB) Index. It is a function of the ratio of the sum of within-cluster scatter to the inter-cluster separation. The scatter within the $i^{t h}$ cluster $S_{i}$ is computed as:

$$
S_{i}=\frac{1}{N_{i}} \sum_{x \in C_{i}}\left|x-\mu_{i}\right|
$$

and the distance between two clusters $C_{i}$ and $C_{j}$, denoted by $D_{i j}$, is defined as $D_{i j}=\left|\mu_{i}-\mu_{j}\right|$ such that $\mu_{i}$ represents the $i^{\text {th }}$ cluster center. The DB index is then defined as:

$$
D B=\frac{1}{k} \sum_{i=1}^{k} R_{i, q l}
$$


where

$$
R_{i, q l}=\max _{j, j \neq i}\left\{\frac{S_{i, q}+S_{j, q}}{D_{i j, l}}\right\}
$$

and the objective to minimize the DB index for achieving proper clustering.

\subsection{Similarity Measurement}

Distance functions are used to measure similarity or dissimilarity of two feature vectors. In a $d$-dimensional space, for any two elements $x$ and $y, D(x, y)$ is a real number and represents the distance between them and is called a distance function. There are many distance functions such as Manhattan, Euclidean, Minkowski and the Mahalanobis. In our scheme we have compared performance of the Euclidean distance and the Mahalanobis distance functions. If $x$ and $y$ are the two feature vectors of same dimension $d$, then these two distance functions are represented by Equation (11) and Equation (12), respectively as:

$$
\begin{gathered}
D(x, y)=\left(\sum_{i=0}^{d-1}\left(x_{i}-y_{i}\right)^{2}\right)^{\frac{1}{2}}=\sqrt{(x-y)^{t}(x-y)} \\
D(x, y)=\left[(x-y)^{t} \Sigma^{-1}(x-y)\right]^{\frac{1}{2}}
\end{gathered}
$$

where $\Sigma$ is the covariance matrix of $x$ and is given as:

$$
\Sigma=\frac{1}{N} \sum_{i=0}^{N-1}\left(x_{i}-\mu\right)\left(x_{i}-\mu\right)^{t} \text { and } \mu=\sum_{i=0}^{N-1} x_{i}
$$

$\Sigma$ is a positive, semi-definite and symmetric matrix. When $\Sigma=I$, the distance is reduced to the Euclidean distance and when $\Sigma \neq I$, it is called the Mahalanobis distance. Both the Euclidean and the Mahalanobis distance functions are commonly used in clustering algorithms. Generally, center of a cluster is determined by its mean vector $\mu$ and its shape is determined by its covariance matrix $\Sigma$.

\subsection{Neural Network}

After clustering, we use neural network as part of the retrieval engine. The neural network in our scheme consists of 3 layers such that there are 7 nodes in the input layer and the number of nodes in the output layer are same as the number of clusters $k$. Our choice of a neural network design is based on a study of decision regions provided in [13. This study demonstrates that a 3 layer network can form arbitrarily complex decision regions and can also provide some insight into the problem of selecting the number of nodes in the 3-layer networks. The number of nodes in the second layer must be greater than 1 when decision regions are disconnected or meshed and cannot be formed from one convex area. In worst case, the number of nodes required in second layer are equal to the number of 
disconnected regions in the input distributions. The number of nodes in the first layer must typically be sufficient to provide three or more edges for each convex area generated by every second-layer node. There should typically be more than three times as many nodes in the second layer as in the first layer [13. In our approach, moment invariants or Zernike moments of a query image form an input feature vector to the neural network whereas its output determines the best representation of the query image features among the $k$ clusters.

\section{Experimental Results}

We have performed experiments with more than 10,000 binary images, obtained from "A Large Binary Image Database" 14 and "Amsterdam Library of Object Images (ALOI)" [15]. The images in our data set have many variations of same shape and object. The data set consists of some groups of similar shapes that are scaled, rotated and slightly distorted as well as some images that are unique.

We use Davies-Bouldin (DB) Index to evaluate the results of clustering using different values of $k$ as shown in Fig. 2 for the two distance functions. From this figure, one can observe that for smaller values of $k$, e.g., 3 or 4 , clustering results with both of these functions are similar. However, when $k \geq 5$, Mahalanobis distance performs better than the Euclidean distance. It is primarily due to the fact that the Mahalanobis distance presents a precise shape of the cluster whereas the Euclidean distance forms a circle irrespective of the shape of the data set.

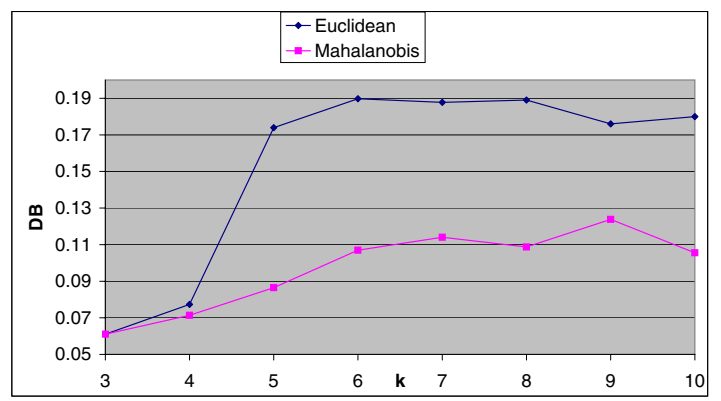

Fig. 2. DB index Vs $k$-means clustering for the two distance functions

For Mahalanobis distance in $k$-means clustering, after assigning samples to the cluster of the nearest mean, we have to re-compute not only the new cluster mean but also the new cluster covariance in each loop of our computation. For Euclidean distance, even after few iterations, it is easy to get a stable cluster means but for Mahalanobis distance, it always takes much longer despite the fact that we use a threshold as a termination condition (new cluster means doesn't move very much, say less than $0.1 \%$ ).

Fig. 3 and Fig. 4 shows the precision-recall graph for our retrieval results. In one set of experiments, the query image chosen was part of the database 


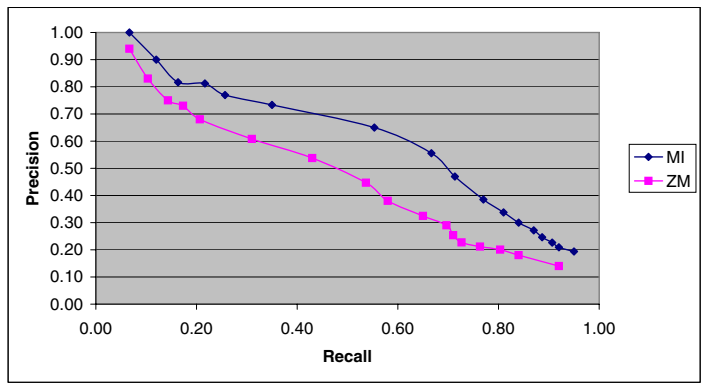

Fig. 3. Precision-recall results when the query image is part of the image database

whereas in some other cases, it was not part of the training sample. The reason for such experiments is to evaluate the system performance in instances when the system has already encountered a similar shape during its training and those when the system has not encountered a similar shape before. As one can observe from these figures, when the query image is not part of the training set, the shape retrieval precision using moment invariants is only $82 \%$ whereas in cases when the query image is part of the training sample, it is about $100 \%$. The precision using Zernike moments in the two cases is only about $72 \%$ and a little more than $90 \%$ respectively. However, in both cases, system was able to retrieve more than $90 \%$ of the relevant images. The reduced recall in both cases can be attributed to the possible clustering problem when the system places similar images in different clusters. Further, it can also be observed that the moment invariants perform better than the Zernike moments for both the precision and the recall. This is partly due to the fact that the Zernike moments have very large coefficients that need to be normalized at the expense of reduced precision.

Images in all of the above sets of experiments reported here contain only a single object. However, we have performed similar experiments on a small data set containing images with multiple objects and obtained comparable results. We have also performed experiments on same images with different sizes and observed that the size of an image doesn't effect either the precision or the recall. All of the images in the experiments reported here are black-and-white, thus, making it an artificial requirement. However, it is important to note that

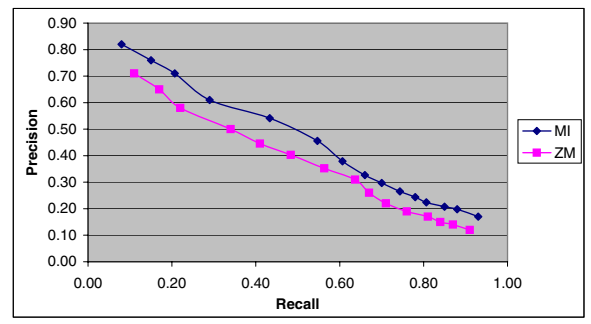

Fig. 4. Precision-recall results when the query image is not part of the image database 


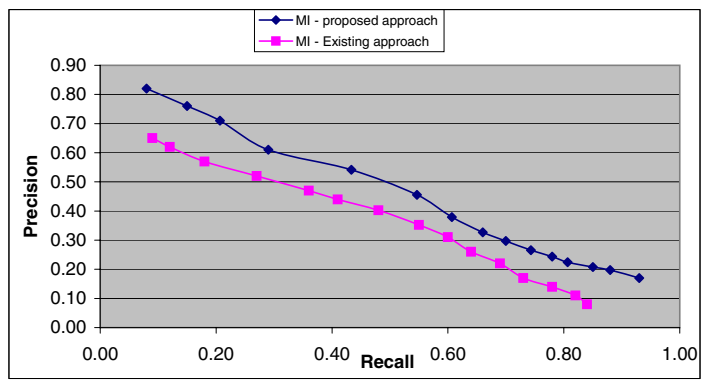

Fig. 5. Comparison of the precision-recall results with the technique proposed in [16]

we are not trying to recognize shapes but only matching on the basis of shape. In practice, many of the image processing and computer vision techniques simply do not take into account the color information.

We have compared the precision-recall results of the proposed technique with the one proposed in [16] and the results are shown in Fig. 5 . Although this technique uses moment invariants to form a feature vector, but feature vectors are managed with the help of $k$-means clustering tree (KCT) in which the value of $k$ determines the number of branches in each node of the tree whereas pointers to the actual images are stored in the leaf nodes. We have chosen this scheme for comparison due to its similarity with our approach as both involve $k$-means clustering to manage similar images and also use neural network as part of the search engine.

We realize that the cost of clustering images and training of neural network can be very high since it involves extensive training and mathematical computations but it is important to realize that images are stored in the database only once and this can be an off-line process to provide retrieval efficiency.

\section{Conclusions and Future Directions}

In this paper, we have used moment invariants and Zernike moments as shape descriptors which can uniquely represent a shape. We have proposed use of $k$ means clustering to organize images and that of neural network as a retrieval engine. Although the training of a neural network is a time consuming process, the training and retrieval are not symmetric and once training is done, it can achieve higher retrieval efficiency and lower computational cost. To refine retrieval results further, there is a need to incorporate relevance feedback and is an important aspect of our future work.

\section{References}

1. Jain, R.: Sf workshop on visual information management system: Workshop report. In: Storage and Retrieval for Image and Video Databases SPIE, vol. 1908, pp. 198-218 (1993)

2. Loncaric, S.: A survey of shape analysis techniques. Pattern Recognition 31(8), 983-1001 (1998) 
3. Hu, M.K.: Visual pattern recognition by moment invariants. IEEE Transaction on Information Theory 8(2), 179-187 (1962)

4. Teh, C.-H., Chin, R.: Image analysis by the methods of moments. IEEE Transacfion on Pattern Analysis and Machine Intelligence 10(4), 496-513 (1988)

5. Maitra, S.: Moment invariants. Proceedings of the IEEE 67, 697-699 (1979)

6. Pavlidis, T.: Survey: A review of algorithms for shape analysis. Computer Graphics Image Processing 7(2), 243-258 (1978)

7. Dai, M., Baylou, P., Najim, M.: An efficient algorithm for computation of shape moments from run-length codes or chain codes. Pattern Recognition 25(10), 1112 1128 (1992)

8. Chen, X., Ahmad, I.: Neural network-based shape retrieval using moment invariants and zernike moments. Technical report 06-002, School of Computer Science, University of Windsor (January 2006)

9. Zhang, D.S., Lu, G.: A comparative study of three region shape descriptors. In: Proceedings of the Sixth Digital Image Computing and Applications (DICTA 2002), pp. 86-91 (January 2002)

10. Teague, M.: Image analysis via the general theory of moments. Journal of Optical Society of America 70(8), 920-930 (1980)

11. Pitas, I.: Digital Image Processing Algorithms. Prentice-Hall, Englewood Cliffs (1993)

12. Zakaria, M., Vroomen, L., Zsombor-Murray, J., van Kessel, H.: Fast algorithm for the computation of moment invariants. Pattern Recognition 20(6), 639-643 (1987)

13. Lippmann, R.: An introduction to computing with neural nets. IEEE Acoustics, Speech and Signal Processing Magazine 4(2), 4-22 (1987)

14. URL: Laboratory for engineering man/machine systems LEMS - a large binary image database (2006), http://www.lems.brown.edu/ dmc

15. Guesebroek, J.M., Burghouts, G., Smeulders, A.: The amsterdam library of object images. International Journal of Computer Vision 61(1), 103-112 (2005)

16. Ahmad, I.: Image indexing and retrieval using moment invariants. In: Proceedings of the $4^{\text {th }}$ iiWAS), Indonesia, pp. 93-104 (September 2002) 